\title{
Processing of Graphic Information in the Study of the Microhardness of the Sintered Sample of Chromium-containing Waste
}

\author{
E.V. Ageev ${ }^{1}$, S.V. Khardikov ${ }^{1}$ \\ ${ }^{1}$ Southwest state University, Kursk, Russia
}

\begin{abstract}
The results of graphic information processing and investigation of the microhardness of the sintered sample from chromiumcontaining waste are presented. Currently, one of the main directions of development of engineering technology is the improvement of existing and the development of new waste-free, environmentally friendly, material-saving production processes. Powder metallurgy is a branch of technology, including the manufacture ofpowders from metals and their alloys and the preparation of blanks and products from them without melting the main component. In most cases, new materials are created in order to provide the optimal combination ofproduct price and operational characteristics. Microhardness is the resistance to plastic indentation (usually on a flat surface) of a solid tip in the shape of a cone or a pyramid made of diamond. With the help of microhardness, they control very small parts, test and sort out watch, instrument and other products. The aim of the work was to study the microhardness of a sintered sample.
\end{abstract}

Keywords: graphic information, sintered samples, chrome, spark-erosion powders.

\section{Introduction}

One of the main directions of development of engineering technology at the present time is the improvement of existing and the development of new waste-free, environmentally friendly, material-saving production processes, that is, those processes that provide blanks with minimal allowances for subsequent machining or without them at the same time reducing consumption of scarce materials. In solving this problem, a specific role belongs to powder metallurgy.

Powder metallurgy is a branch of technology, including the manufacture of powders from metals and their alloys and the preparation of blanks and products from them without melting the main component. Using powder metallurgy methods, it is possible to create materials from various components with sharply differing properties and melting points, new materials with a diverse set of physicomechanical properties. Powder metallurgy is used both to create fundamentally new materials and products from them, and to manufacture the widest range of structural parts for general purposes [1-6].

In most cases, new materials are created in order to provide the optimal combination of product price and operational characteristics. The obtained results contribute to the introduction of powder metallurgy technologies into production, since this simplifies the technology for producing products, reduces material loss, and can significantly improve the characteristics of products, one of which is microhardness.

Microhardness is the resistance to plastic indentation (usually on a flat surface) of a solid tip in the shape of a cone or a pyramid made of diamond. Much less often, microhardness tests are carried out by scratching. The difference in microhardness tests from conventional hardness measurements is very small values of pressing loads and small depth and dimensions of the print.

With the help of microhardness, they control very small parts, test and sort out watch, instrument and other products. The study of the microhardness of rare and noble metals is possible on quantities not sufficient for the manufacture of a sample for testing for ordinary hardness. The fragility of surface layers, coatings (for example, chromium plating) and very fragile materials can be estimated by the number and nature of the location of cracks around the indent [7-10].

The aim of this work was to conduct research on the sintering of powders, obtained by electroerosive dispersion of chromiumcontaining wastes, and to study the microhardness of sintered samples.

\section{Materials and techniques}

To obtain powder from ball-bearing steel wastes by the method of electroerosive dispersion, we used a device for EED of conductive materials developed by the authors of [11- 15] and ballbearing steel wastes. Waste was loaded into a reactor filled with a working fluid - distilled water; the process was carried out with the following electrical parameters: capacitance of discharge capacitors $65 \mathrm{pF}$, voltage 150-170 V, pulse repetition rate 110-120 Hz. As a result of local exposure to short-term electrical discharges between the electrodes, the waste material was destroyed with the formation of dispersed powder particles. The EED process is the destruction of conductive material as a result of local exposure to short-term electrical discharges between the electrodes. In the discharge zone, under the influence of high temperatures, heating, melting, and partial evaporation of the material occur. To obtain high temperature in a limited area of small volume, a large concentration of energy is required. Achieving this goal is carried out using pulsed voltage, and EED is carried out in a liquid medium (working fluid), which fills the gap between the electrodes, called the interelectrode gap.

Due to the fact that any smooth surface has its own macro- or microrelief, there will always be two points between two electrodes, the distance between which will be less than between other points of the electrode surfaces. When a source of electric current is connected to the electrodes, a current begins to flow between the electrodes, and an electric field appears, the intensity of which in the area where the gap between the electrodes is minimal will reach its maximum value. The location of this section depends on local protrusions, irregularities on the electrodes, on the presence and size of electrically conductive particles located in the interelectrode gap. To describe the processes occurring during an electric discharge, we will divide them into three stages.

The first stage of the EED process is the breakdown of the interelectrode gap (formation of a discharge channel) as a result of the formation of a zone with high field strength. Under the action of the discharge, the working fluid is ionized in the gap through which electric current begins to flow. The second stage is the formation of a gas bubble near the discharge channel from liquid and metal vapor, and heating of the electrodes. The development of a discharge in the interelectrode gap is accompanied by the appearance of a shock thermal wave, which occurs in the initial stages of the discharge and leads to the formation of a gas bubble in the working fluid. The third stage will be the cessation of current, separation of the shock wave from the gas bubble and the continuation of its expansion by inertia, the evacuation of erosion products.

To study the properties of the obtained powder material, it was sintered. One of the properties of a sintered sample is microhardness. The hardness of a material means its resistance to local plastic deformation when another body penetrates into it that is not subject to such deformation. From the definition it follows 
that the hardness of the implanted body must exceed that of the test sample by a sufficient amount. Consider the definition of hardness by the Vickers method.

All methods differ both in measurement technology and in the tools and devices used. The most common three methods for determining the value of hardness:

Resistance to indentation of a solid tip (indenter) in the test sample. The shape of the indenter can be very diverse - in the form of a cone, ball, pyramid, etc.

Scratch resistance with high strength reference samples.

Determination of the rolling resistance of the pendulum, where the test body is the support of the tip of a certain shape. Most measurements are carried out on a flat surface of the test material. Each of the methods for determining technical characteristics gives a certain error. For greater accuracy, compliance with the conditions for the size of the test sample, its minimum thickness, is required. In addition, each technique works only in a certain range of measurement values. The method of determining Vickers hardness is based on the study of the dependence of the penetration depth of the diamond cone (indenter) into the material under study on the force magnitude. After removing the force, an imprint remains on the surface of the sample corresponding to the immersion depth of the indenter. Due to the fact that the geometrical dimensions of the indenter are known and strictly regulated, instead of immersion depth, the imprint area in the surface layer of the test material is determined.

Vickers hardness testing is possible for substances with the highest values, since a diamond pyramid is used as a test cone, which has the maximum known hardness.

The indenter is made in the form of a quadrangular pyramid with angles between the faces of $136^{\circ}$. This angle was chosen in order to bring the values of the Vickers method closer to the Brinnel method. Thus, the hardness values in the range of 400-450 units practically coincide, especially in the region of lower values. Vickers hardness is determined by pressing a pyramid into a test sample under the action of a certain magnitude force. Knowing the applied force and the area of the print, it is possible to determine the surface hardness of the test material.

Instead of calculating the imprint area, the values of the measured rhombus diagonals are used, between which there is a direct relationship.

The final hardness result is determined by the formula:

$\mathrm{HV}=1.854-\mathrm{F} / \mathrm{d}^{2}$

In this formula, $\mathrm{F}$ - the value of force, and $\mathrm{d}$ - the diagonal of the rhombus.

The magnitude of the load depends on the material being measured (its estimated hardness). As harder the surface of the test material sample, as greater the load. This is due to the desire to reduce the error in determining the area and reduce the influence of the viscosity of the material.

To reduce the error, restrictions are also imposed on the size of the test sample. The minimum thickness of the sample should be in 1.2-1.5 times larger than the expected diagonal of the print depending on the type of metal (a smaller value corresponds to steel, a larger one is for non-ferrous metals). The distance between the edge of the sample or the edge of the previous print and the center of the print should be at least 2.5 diagonal values.

Special requirements are also imposed on surface cleanliness. Its roughness should not exceed 0.16 microns, which means the need for polishing the surface. The small linear dimensions of the sample require the use of a microscope to measure the size of the print, and as harder the sample, as more clear the picture must transmit the microscope to maintain measurement accuracy.

Vickers hardness measurement is a universal method, but it gives the most accurate values when studying substances with high hardness. Small efforts and, consequently, small linear dimensions of the print allow practically not violating the surface of the measured material.

Vickers microhardness is determined by measuring the diagonals of the imprint, obtained in the test surface by indenting a diamond tetrahedral pyramid with an angle at the apex of $\mathrm{a}=$ $136^{\circ}$. The values of the diagonals are measured on a microscope. The main parameters for determining Vickers hardness are: load $30 \mathrm{kgf}$, holding time for steels $-10 \ldots 15 \mathrm{~s}$, the dimension of the hardness number $\left(\mathrm{kgf} / \mathrm{mm}^{2}\right.$ ) is not set. Например, $420 \mathrm{HV}$ означает твёрдость, полученную при этих параметрах. If the parameters differ from the main ones (for non-ferrous metals, the indenter exposure time for a load is $30 \mathrm{~s}$ ), then, for example, 450 HV10 / 15 are recorded - it means that the Vickers hardness number 450 is obtained at a load of $10 \mathrm{kgf}(98.1 \mathrm{~N})$ applied to diamond pyramid for $15 \mathrm{~s}$.

\section{Conduct}

Quanta 3D DualBeam microscope-FEI's most versatile microscope for 2D and 3D characterization and material analysis, Quanta 3D has three SEM imaging modes (high vacuum, low vacuum, and ambient mode) to enable you to match characteristics to a wide range of samples. The built-in focused ion beam (FIP) adds the possibility of obtaining cross-sections of the sample, which extends the range of application of the microscope. Natural environment mode allows in situ study of the dynamic behavior of materials at different levels of humidity, temperature and pressure.

Quanta 3D DualBeam (scanning electron microscope combined with focused ion beam system) opens up new possibilities and flexibility for engineers and researchers who need to characterize materials, analyze faults and control processes. The system combines traditional thermal scanning electron microscopy (SEM) with focused ion beam (FIP) in addition to the existing instruments in your laboratory and extends the range of applications for 3D research and nanoanalysis, TEM sample preparation or structural modification of the sample surface at the nanometer scale. (figure 1).

The Quanta 3D DualBeam microscope is a combination of two systems:

- scanning electron microscope (SEM), giving images of a variety of samples in digital form with a magnification of more than 100000 times;

- focused ion beam (FIB), able to quickly and precisely remove the layer of the sample material, to expose the structure under the surface layer, create a section, place the layer of material, etc. in addition, ion beam, as well as electronic, can create a high resolution image.

The integration of both systems in one instrument forms a powerful analytical tool capable of analyzing a wide variety of samples in three-dimensional space. Switching between the two beams allows, on the one hand, fast and accurate navigation, and, on the other, the possibility of precision removal of layers of material. The combination of electron and FIP beams on a short working segment allows the analysis to be carried out in the "cut and saw" mode with high resolution. The workstation provides optimal performance, resolution, and automation. In addition, the combination of FIP and SEM technologies in one device adds new possibilities to the microscope:

- creation of electron beam images of sections created with the help of FIP without erosion of the area of interest;

- real-time acquisition by electron beam of individual frames and films simultaneously with removal of material layers by FIP;

- removal of the charge by the electron beam during removal of the material using FIP;

- microanalysis of defect cross-section elements;

- imaging of the sample surface by electron beam without erosion or parasitic implantation of gallium ion beam;

- preparation of samples for transmission electron microscope (TEM).

Basic advantages:

Increase visualization and analysis capabilities: capture surface and volume information and reconstruct image and 
analytical data in three dimensions

Increase throughput and explore larger areas with high current Quanta FIB for rapid removal (bleed) of material

Empowerment in the field of nanocharacterization using treatment $(2 \mathrm{kV})$ PAM / nuclear probe / EBSD samples; removing the amorphous regions without cleaning with argon Improvement of registration and nanopatterned nonconductive samples in the "neutralization of the charge"

Increase throughput for your samples with an automated system for cutting FIP or preparing TEM samples

The ability to work with a wide range of materials, which is provided by the presence of a large number of detector settings, flexibility in setting up the system for samples of non-standard shape or advanced experiments

Increased flexibility in the characterization of samples in a dynamic environment; keeping samples wet or heating them for ESEM experiments

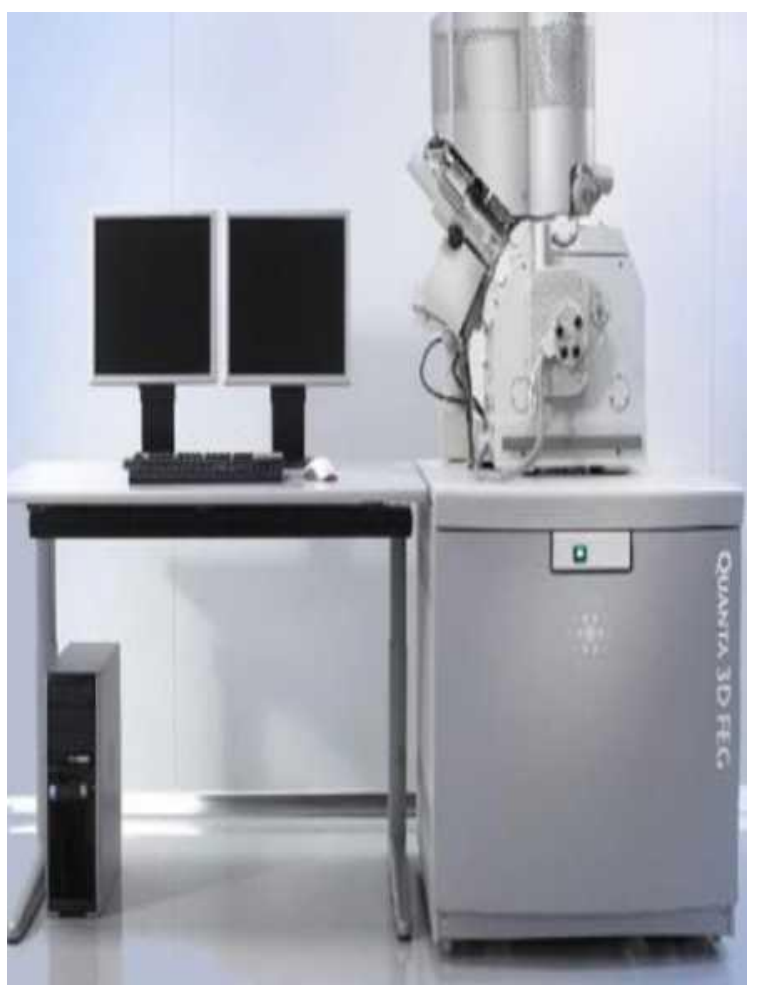

Fig. 1. Quanta 600 FEG microscope

Hardness tests of the surface and transverse sections were carried out using the DM-8 automatic microhardness analysis system by the Micro-Vickers method with an indenter load of 50 $\mathrm{g}$ using ten imprints with a free injection site in accordance with GOST 9450-76 (Measuring microhardness by indentation of diamond tips ). The indenter loading time was $15 \mathrm{~s}$. The measurement results are shown in table 1 .

The results of the study of the sintered chromium- containing sample microstructure, performed using a Quanta 600 FEG scanning electron microscope, are presented in Figure 2.

From image analysis 2 it was found that the sintered sample consists of spherical particles, while small particles fill the voids between the large ones, therefore it is not very porous. This allows you to more accurately determine the cope of the sintered products obtained.

From the analysis of the image of the microstructure of the sintered sample it can be seen that the sintered particles have an equilibrium shape, the boundaries between the particles are thinned, and their continuity is broken. This leads to the appearance of qualitatively new contact areas with characteristic metallic properties.

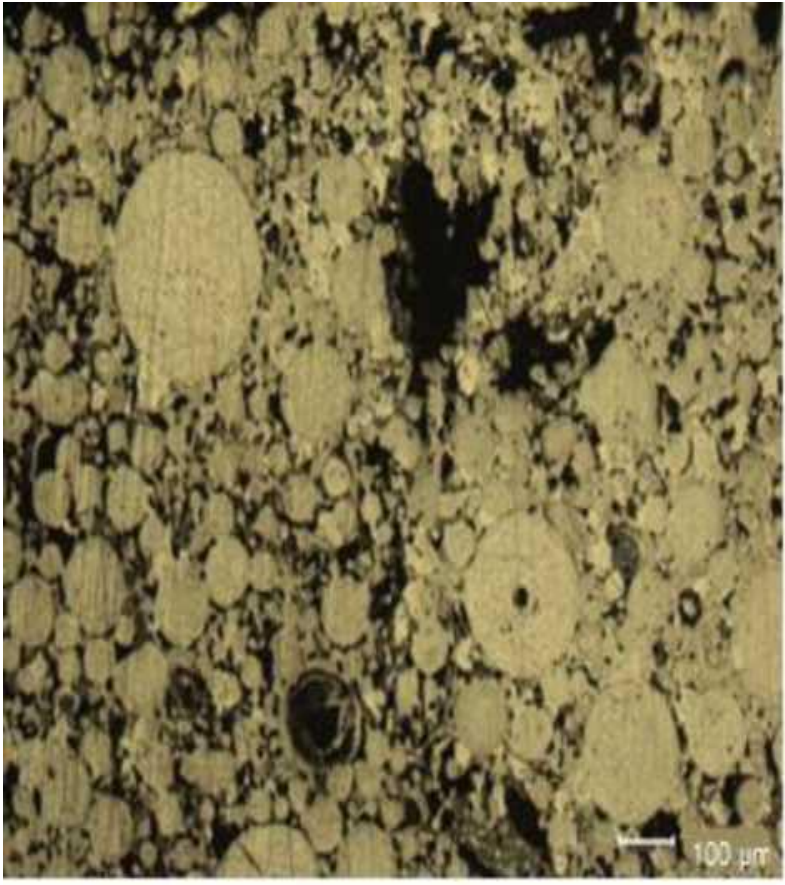

Fig. 2. Photo of cross section of sintered specimen

Table 1. Vickers microhardness

\begin{tabular}{cc}
\hline Measurement number & HV value at $50 \mathrm{~g}$ load \\
\hline 1 & 752 \\
3 & 746 \\
4 & 741 \\
5 & 739 \\
6 & 743 \\
7 & 744 \\
8 & 749 \\
9 & 877 \\
10 & 736 \\
Average value & 712 \\
\hline
\end{tabular}

The spread of microhardness values over the surface is associated with an uneven distribution of particles in the sintered sample.

Figure 3 shows a snapshot of one of the injection site with a diamond tip.

In image analysis it was established that there is an increase in the hardness of sintered samples with an increase in sintering temperature. With increasing sintering temperature, the density and strength of the sintered products increase as faster as lower the pressing pressure. At low temperatures, shrinkage is negligible, since moisture evaporates, adsorbed gases are removed, and surface oxides are reduced. In the region of high temperatures, a significant increase in the metal contact between the particles occurs, the pores become denser under the action of surface tension forces and the compression shrinks. From the analysis of the image of the microstructure of the sintered sample it can be seen that the sintered particles have an equilibrium shape, the boundaries between the particles are thinned, and their continuity is broken. This leads to the appearance of qualitatively new contact areas with characteristic metallic properties 


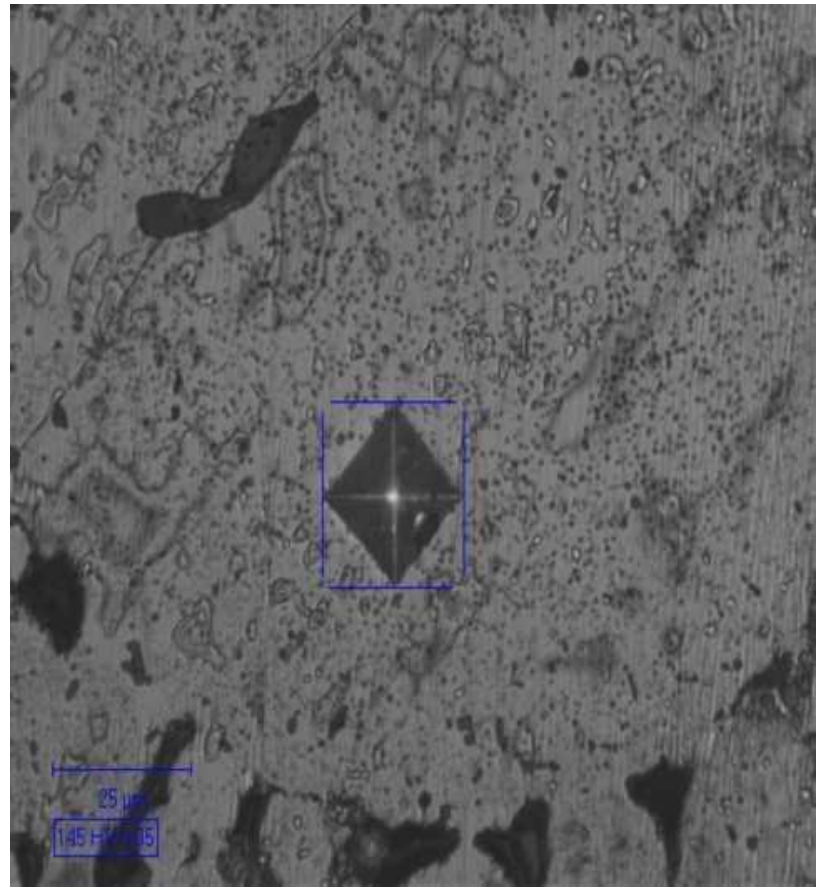

Fig. 3. Photo of the indentation point of the tip

Thus, a study of the hardness of sintered samples confirms that a lower fineness of the chromium-containing powder allows for greater hardness at a lower sintering temperature.

\section{Conclusion}

The results of the work are as follows. According to the results of studies of images of sintered samples of chromium- containing steel, conducted on the microscope Quanta 600 FEG, it was found that microhardness of sintered samples from powders, obtained by electroerosive dispersion of chromium-containing waste in lighting kerosene, is $754 \mathrm{HV}$. This allows you to determine the scope of the resulting products.

From image analysis it was found that the sintered sample consists of spherical particles, while small particles fill the voids between the large ones, therefore it is not very porous. Thus, the problem associated with the processing and reuse of chromiumcontaining waste can be successfully solved by electroerosive dispersion and subsequent isostatic pressing at a pressure of 250 $\mathrm{MPa}$ and sintering for 2 hours at a temperature of $1250^{\circ} \mathrm{C}$ in argon.

As a result, by processing images from the microscope Quanta $600 \mathrm{FEG}$, it was possible to determine the important parameters of the sintered products obtained from chromium- containing steels obtained carbon-containing working fluid.

\section{Acknowledgments}

The work was supported by a scholarship of the President of the Russian Federation to young scientists and graduate students (SP-945.2019.1).

\section{References}

[1] Ageev E.V., Kirichek A.V., Altuhov A.Yu., Ageeva E.V., J. Nano- Electron. Phys. 6 No 3, 03001 (2014).

[2] Ageev E.V., The patent 2449859, the Russian Federation, C2, B22F9/14. No 2010104316/02; appl. 08.02.2010; publ. 10.05.2012. - 4 p.

[3] Ageeva E.V., Ageev E.V., Horyakova N.M., Malukhov V.S., J. Nano- Electron. Phys. 6 No 3, 03011 (2014).

[4] Ageeva E.V., Ageev E.V., Karpenko V.Yu., Osminina A.S., J. Nano- Electron. Phys. 6 No 3, 03049 (2014).
[5] Chandler K.M., Mitchell M.D., Pikuz S.A., Shelkovenko T.A., Hammer D.A., Shlyaptseva A.S., Ouart N.D., Hansen S.B., Kantsyrev V.L., Fedin D.A., Rev. Sci. Instrum 75 No 10-2, 3702 (2004)

[6] Ershova T.B., Dvornik M.I.,. Zaitsev A.V, Composite. Nanostruct. No 2, 40 (2011).

[7] Falkowski V.A., Falkowski F.I., Panov V.S., NonFerrous Metal. No 10, 85 (2007).

[8] Falkowski V.A., Falkowski F.I.,. Panov V.S, NonFerrous Metal. No 10, 85 (2007).

[9] Gusev, V.N. Anodic-mechanical processing of metals (1952) $321 \mathrm{p}$.

[10] Hertel N., Ferrous Metal. No 2, 50 (2012).

[11] Janitor M.I. Development of physico-chemical and technological fundamentals of the processing of tungsten- cobalt hard alloy by electroerosive dispersion: diss. ... cand. tech. Sciences / Dvornik Maxim Ivanovich. - Khabarovsk, (2006) . - 116 p.

[12] Kazi I.H., Wild P.M.,. Moore T.N, Sayer M., Thin Solid Films 515 No 4, 2602 (2006).

[13] Maslenkov S.B., All materials. Encyclopedic Reference No 1,18 (2007).

[14] Tretyakov V.I., Basics of metallurgy and production technology of sintered hard alloys (Moskow: Metallurgy: 1976).

[15] Wang Z., Qiu K., Electrochem. Commun. 8 No 7, 1075 (2006). 